\title{
Identification of the occurrence and potential mechanisms of heterotopic ossification associated with 17-beta-estradiol targeting MKX by bioinformatics analysis and cellular experiments
}

\author{
Yunpeng Zhang ${ }^{\text {Equal first author, } 1}$, Jingwei Zhang ${ }^{\text {Equal first author, 2, } 3}$, Chenyu Sun ${ }^{4}$, Fan Wu ${ }^{\text {Corresp. } 1}$ \\ ${ }^{1}$ Department of surgery, Shanghai Fengxian District Central Hospital, Shanghai, China \\ 2 Department of Orthopedics, Shanghai Fengxian District Central Hospital, Shanghai, China \\ 3 Shanghai University of Medicine \& Health Sciences Affiliated Sixth People's Hospital South Campus, Shanghai, China \\ 4 Department of Internal Medicine, AMITA Health Saint Joseph Hospital Chicago, Chicago, Illinois, The United States \\ Corresponding Author: Fan Wu \\ Email address: WF131@seu.edu.mk
}

Background: Tendon heterotopic ossification $(\mathrm{HO})$ is a common condition occurring secondary to tendon injury or surgical trauma that significantly affects the patient's quality of life. The treatment of tendon HO remains challenging due to a lack of clarity regarding the pathological mechanism. Mohawk (MKX) is a key factor in preventing tendon $\mathrm{HO}$; however, its upstream regulatory mechanism remains to be understood. This study aimed to identify potential compounds that target and regulate MKX and explore their functional mechanisms

Methods: Bioinformatics analysis of MKX-related compounds and proteins was performed based on data from the STITCH and OncoBinder databases. Subsequently, the SymMap database was used to study MKX-related traditional Chinese medicine drugs and symptoms. Next, the OncoBinder genomic and proteomic discovery model was applied to identify potential regulators of MKX. The analytical tool, Expert Protein Analysis System for proteomics was used to predict the three-dimensional structure of MKX, and the AutoDockTools software was used to identify pockets of activity at potential sites for molecular docking. Furthermore, we evaluated the effect of different doses of 17-beta-estradiol on bone marrowderived mesenchymal stem cells (BM-MSCs).

Results: By predicting the three-dimensional structure of MKX and simulating molecular docking, Pro-Tyr and 17-beta-Estradiol were found to target and bind to MKX. Analysis of the STITCH and OncoBinder databases showed that MKX had a significant regulatory correlation with suppressor interacting 3 A/histone deacetylase 1 (SIN3A/HDAC1). The GO and KEGG pathway enrichment analysis revealed that the functions of MKX and its associated proteins were mainly enriched in osteogenic-related pathways. Assessment of the proliferation of BM-MSCs revealed that 17-beta-estradiol possibly upregulated the mRNA expression of the HDAC1-SIN3A/BMP pathway-related RUNX2, thereby promoting the proliferation of BM-MSCs.

Conclusions: The compounds Pro-Tyr and 17-beta-Estradiol may bind to MKX and thus affect the interaction of MKX with SIN3A/HDAC1. 
1 Original

2 Identification of the occurrence and potential mechanisms of heterotopic ossification associated with 17-beta-estradiol targeting MKX by bioinformatics analysis and cellular

4 experiments

5 Running title: Potential mechanisms of HO

6 Yunpeng Zhang ${ }^{1 \#}$, Jingwei Zhang ${ }^{2,3 \#}$, Chenyu $\mathrm{Sun}^{4}$, Fan $\mathrm{Wu}^{1}$

7 Department of surgery, Shanghai Fengxian District Central Hospital

$8 \quad{ }^{2}$ Department of Orthopedics,Shanghai Fengxian District Central Hospital;

$9{ }^{3}$ Shanghai University of Medicine \& Health Sciences Affiliated Sixth People's Hospital South

10 Campus, Shanghai 201499,China;

$11{ }^{4}$ Department of Internal Medicine, AMITA Health Saint Joseph Hospital Chicago, 2900 N. Lake

12 Shore Drive, Chicago 60657, Illinois, USA

13 \#These authors contributed equally to this work

\section{*Corresponding Author}

16 Fan $\mathrm{Wu}$

17 Department of surgery, Shanghai Fengxian District Central Hospital.

18 Tel: 18817518830

19 Email: WF131@seu.edu.mk 


\section{ABSTRACT}

Background: Tendon heterotopic ossification (HO) is a common condition occurring secondary to tendon injury or surgical trauma that significantly affects the patient's quality of life. The treatment of tendon $\mathrm{HO}$ remains challenging due to a lack of clarity regarding the pathological mechanism. Mohawk (MKX) is a key factor in preventing tendon HO; however, its upstream regulatory mechanism remains to be understood. This study aimed to identify potential compounds that target and regulate MKX and explore their functional mechanisms

Methods: Bioinformatics analysis of MKX-related compounds and proteins was performed based on data from the STITCH and OncoBinder databases. Subsequently, the SymMap database was used to study MKX-related traditional Chinese medicine drugs and symptoms. Next, the OncoBinder genomic and proteomic discovery model was applied to identify potential regulators of MKX. The analytical tool, Expert Protein Analysis System for proteomics was used to predict the three-dimensional structure of MKX, and the AutoDockTools software was used to identify pockets of activity at potential sites for molecular docking. Furthermore, we evaluated the effect of different doses of 17-beta-estradiol on bone marrow-derived mesenchymal stem cells (BMMSCs).

Results: By predicting the three-dimensional structure of MKX and simulating molecular docking, Pro-Tyr and 17-beta-Estradiol were found to target and bind to MKX. Analysis of the STITCH and OncoBinder databases showed that MKX had a significant regulatory correlation with suppressor interacting $3 \mathrm{~A} /$ histone deacetylase 1 (SIN3A/HDAC1). The GO and KEGG pathway enrichment analysis revealed that the functions of MKX and its associated proteins were mainly enriched in osteogenic-related pathways. Assessment of the proliferation of BMMSCs revealed that 17-beta-estradiol possibly upregulated the mRNA expression of the 
43 HDAC1-SIN3A/BMP pathway-related RUNX2, thereby promoting the proliferation of BM-

44 MSCs.

45 Conclusions: The compounds Pro-Tyr and 17-beta-Estradiol may bind to MKX and thus affect

46 the interaction of MKX with SIN3A/HDAC1.

47 Key words: MKX, Tendon HO, SIN3A, HDAC1, 17-beta-Estradiol, Pro-Tyr. 


\section{Abbreviations}

52 American Type Culture Collection (ATCC); false discovery rate (FDR); Gene Set Enrichment

53 Analysis (GSEA); Bone Marrow-Derived Mesenchymal Stem Cells (hBMSCs); heterotopic

54 ossification (HO); Mohawk (MKX); modern medicine (MM);

55 Mesenchymal Stem Cells (MSCs); Mesenchymal Stem Cell Medium (MSCM); optical density

56 (OD); Runt-related Transcriptional Factor 2 (RUNX2); Suppressor interacting 3a (Sin3a);

57 traditional Chinese medicine (TCM); Transforming Growth Factor-beta 1 (TGF-beta1).

58

59

60

61

62

63

64

65

66

67

68

69

70

71

72

73 
75

\section{Introduction}

Tendons are connective tissues that connect muscles and bones. Tendons play a role in transmitting muscle contractions to the bone and are highly susceptible to injury during exercise (Giddings et al., 2000). Statistics reveal that more than 16 million people in the United States suffer from key muscle injuries each year (James et al., 2008). Tendon heterotopic ossification (HO) is a common condition occurring secondary to tendon injury or surgical trauma, which can lead to varying degrees of pain, edema, decreased mechanical properties of the tendon or even rupture, and limited joint movement that can significantly affect the patient's quality of life. The treatment of tendon $\mathrm{HO}$ remains a major challenge due to a lack of clarity regarding the pathological mechanism (O'Brien et al., 2012). Therefore, a better understanding of the pathological mechanism will provide new therapeutic targets and ideas for the treatment of tendon $\mathrm{HO}$.

Recent studies have shown that knockdown of the tendon-specific transcription factor Mohawk (MKX) leads to ectopic tendon ossification, suggesting that MKX may be a key factor in preventing the ossification (Suzuki et al., 2016; Liu, Xu \& Jiang, 2019). MKX is essential for the development and differentiation of tendons (Ito et al., 2010; Liu et al., 2010, 2015; Kimura et al., 2011; Suzuki et al., 2016). In vitro culture of MKX-knockdown rat tendon-derived cells and induction of differentiation showed that the knockdown accelerated chondrogenesis and osteogenic differentiation, while overexpression of MKX inhibited chondrogenesis, osteogenesis, and adipogenic differentiation (Suzuki et al., 2016). These results suggest that MKX has a dual function, namely maintaining the normal differentiation of tendon cells and inhibiting the expression of chondrogenesis-related genes, thus suppressing the transdifferentiation of tendon 
97 and ligament cells to other lineages. Currently, there is limited understanding of the upstream 98 regulatory mechanism of $\mathrm{MKX}$.

99 In the STITCH database, each compound-protein interaction corresponds to a score 100 indicating the probability of interaction and affinity between them(Szklarczyk et al., 2016). We

101

102

103

104

105

106

107

108

109

110

111

112

113

114 115

116

117

118

119 used data from the STITCH (http://stitch.embl.de) database to identify MKX-related compounds and proteins. SymMap database was used in this study to examine the MKX-related herbal drugs and symptoms (Wu et al., 2019); OncoBinder genomic and proteomic discovery models were used to identify potential regulators of MKX (Van Coillie et al., 2016). The Expert Protein Analysis System (ExPASy) is a specialized proteomics analysis tool that combines information from several databases, including SWISS-PROT, TrEMBL, ENZYME, PROSITE, and the SWISS-MODEL repository (Gasteiger, 2003). ExPASy was used to predict the structure of MKX in this study, and AutoDockTools software was used for molecular docking. The effects of different doses of 17- $\beta$-estradiol on bone marrow mesenchymal stem cells (BM-MSCs) were also evaluated.

We speculated that MKX could maintain normal differentiation of tendon cells and inhibit the expression of genes related to cartilage formation. Therefore, this study aimed to identify potential molecules that target and regulate $\mathrm{MKX}$, reveal the mechanism by which $\mathrm{MKX}$ contributes to the development of HO, identify novel effective therapeutic targets, and improve the diagnosis and prognosis of patients with $\mathrm{HO}$.

\section{Materials and Methods}

\section{Bioinformatics analysis of MKX and Structure prediction of MKX}

We used data from the STITCH (http://stitch.embl.de) database to identify MKX-related 
120 compounds and proteins (Szklarczyk et al., 2016). We used data from the SymMap

121 (https://www.symmap.org/) database to identify MKX-related traditional Chinese medicine

122 (TCM) drugs and symptoms (Wu et al., 2019). We also applied a genomic and proteomic

123 discovery model named OncoBinder to identify potential regulators of MKX (Van Coillie et al.,

124 2016). The tool was used to predict the structure of MKX (Gasteiger, 2003).

125

126 Enrichment analysis

127 The GO and KEGG pathway enrichment analysis was performed as described in the citation 128 and visualized by $\mathrm{R}$ software ( $\mathrm{Yu}$ et al., 2012). The clusterProfiler package is used for 129 enrichment analysis, while the org.Hs.eg.db package is used for ID conversion. Gene Set 130 Enrichment Analysis (GSEA) analysis was performed using the clusterProfiler package of R 131 software [version 3.14.3] (Subramanian et al., 2005). The reference gene collection used by 132 GSEA is c2.cp.v7.2.symbols.gmt, and the gene set database used is MSigDB collections. 133 Conditions were considered significantly enriched when the false discovery rate was $<0.25$ and 134 p.adjust was $<0.05$.

\section{Molecular docking analysis}

137 Molecular docking analysis is an important method for predicting compound and protein 138 binding (Friesner et al., 2004; He et al., 2019; Mehta, Miszta \& Filipek, 2021). The structures of 139 Pro-Tyr and 17-beta-estradiol were searched in the PubChem database. After the three140 dimensional structure of MKX was predicted by the ExPASy tool, the AutoDockTools software was used to determine the active pockets of potential sites for molecular docking. The structure

142 has been verified and structure minimization has been performed before the docking study. 
143 Finally, the AutoDock Vina software was used to perform molecular docking to verify the 144 targeting of MKX with Pro-Tyr and 17-beta-estradiol. The lowest binding energy values were 145 considered to visualize the results using the PyMOL software.

\section{Molecule dynamics}

The complex system of each compound with the target egg obtained by docking was used as the initial structure to perform all-atom molecular dynamics simulations (MD) separately. The charges of the small molecules were calculated using the antechamber module and the HartreeFock (HF) SCF/6-31G* of the gaussian 09 software. Afterwards, small molecules and proteins were described using force fields (Wang et al., 2004; Maier et al., 2015). The LEaP module was

153

154

155

156

157

used to add hydrogen atoms to the system, a truncated tetrahedral TIP3P solvent cartridge was added at a distance of $10 \AA$ from the system, and $\mathrm{Na}+/ \mathrm{Cl}$ - was added to the system for balancing the system charge.

Molecular dynamics simulations were performed using AMBER 18 software (Lee et al., 2020). After the energy optimization of the system was completed, a 200 ps warming of the system at a fixed volume and constant warming rate was used to slowly increase the system temperature from $0 \mathrm{~K}$ to $298.15 \mathrm{~K}$. Finally, these composite systems are simulated with $30 \mathrm{~ns}$ NPT (isothermal isobaric) tethering under periodic boundary conditions, respectively.For the simulations, the truncation distance of the non-bond was set to $8 \AA$. The Particle mesh Ewald (PME) method was used to calculate the long-range electrostatic interaction, the SHAKE method was used to limit the bond length of hydrogen atoms, and the Langevin algorithm was used for temperature control (Larini, Mannella \& Leporini, 2007; Mehta, Miszta \& Filipek, 2021). Traces were saved at 10 ps intervals for subsequent analysis. 
167

168

169

170

171

172

173

174

175

176

177

178

179

180

181

182

183

184

185

186

187 188

\section{MMGBSA free energy calculation}

The binding free energy between protein and ligand for all systems was calculated by the MM/GBSA method (Genheden \& Ryde, 2015; Chen et al., 2020). The MD trajectory of 25-30 ns was used as the calculation in this study. And the specific equation was as follows:

$$
\begin{aligned}
\Delta G_{\text {bind }} & =\Delta \mathrm{G}_{\text {complex }}-\left(\Delta \mathrm{G}_{\text {receptor }}+\Delta \mathrm{G}_{\text {ligand }}\right) \\
& =\Delta \mathrm{E}_{\text {internal }}+\Delta \mathrm{E}_{\mathrm{VDW}}+\Delta \mathrm{E}_{\text {elec }}+\Delta \mathrm{G}_{\mathrm{GB}}+\Delta \mathrm{G}_{\mathrm{SA}}
\end{aligned}
$$

$\Delta \mathrm{E}_{\text {internal }}$ represents internal energy, $\Delta \mathrm{E}_{\mathrm{VDW}}$ represents van der Waals interaction, and $\Delta \mathrm{E}_{\mathrm{elec}}$ represents electrostatic interaction. $\Delta \mathrm{G}_{\mathrm{GB}}$ and $\Delta \mathrm{G}_{\mathrm{GA}}$ were collectively referred to as the solvation free energy. Where GGB was the polar solvation free energy and GSA was the nonpolar solvation free energy. The GB model developed by Nguyen was used to calculate $\Delta \mathrm{G}_{\mathrm{GB}}$ (Nguyen, Roe \& Simmerling, 2013).

\section{Experimental cells and culture}

The cells used in this study were human, primary bone marrow-derived mesenchymal stem cells (BM-MSCs, Normal, Human, ATCC PCS-500-012), purchased from the American Type Culture Collection (ATCC). BM-MSCs were used, which was supplemented with 7\% Fetal Bovine Serum (FBS), $15 \mathrm{ng} / \mathrm{mL}$ insulin-like growth factor 1 (rhIGF-1), $125 \mathrm{pg} / \mathrm{mL}$ fibroblast growth factor-basic (rhFGF), $2.4 \mathrm{mM}$ L-alanyl-L-glutamine, and 1\% penicillin-streptomycin solution $(\mathrm{P} / \mathrm{S})$. All cells were cultured at $37^{\circ} \mathrm{C}$ in a cell culture incubator with $5 \% \mathrm{CO}_{2}$, and the experiment was completed with five passages of cells. Passaged BM-MSCs $(n=1,000)$ were placed in each well of a 96-well plate, divided into three groups, and were exposed to different doses of 17-beta-estradiol $(0,0.001,0.01 \mathrm{nmol} / \mathrm{L})$. Accordingly, the groups were categorized as control, $0.001 \mathrm{nmol} / \mathrm{L}$ 17-beta-estradiol-treated, and $0.01 \mathrm{nmol} / \mathrm{L}$ 17-beta-estradiol-treated. 
189

190

191

192

193

194

195

196

197

198

199

200

201

202

203

204

205

206

207

208

209

210

211

\section{Real-Time Quantitative Reverse Transcription PCR}

Real-time quantitative reverse tanscription polymerase chain reaction (RT-qPCR) was performed to determine the mRNA expression of runt-related transcription factor 2 (RUNX2). RT-PCR was performed to quantify the expression of genes and cytokines associated with RUNX2 at 1, 3, and $5 \mathrm{~d}$ after the induction. Total RNA was extracted from the BM-MSCs using the total RNA extraction reagent (TRIzol) and purified using the QIAquick PCR purification kit. The first-strand cDNA synthesis kit and random hexamer primers were used for the reverse transcription reaction to obtain the cDNA using DNAse I. The quantitative RT-PCR was performed using a fluorescent quantitative PCR kit (SYBR Green PCR Master Mix). $\beta$-actin was selected as the internal reference (Supplementary table 1). PCR cycling condition was adjusted as follows: $95{ }^{\circ} \mathrm{C}$ for $2 \mathrm{~min}, 94{ }^{\circ} \mathrm{C}$ for $30 \mathrm{~s}, 55^{\circ} \mathrm{C}$ for $30 \mathrm{~s}$, and then at $72{ }^{\circ} \mathrm{C}$ for 1 min with 40 cycles performed. 2-DDCt method was used to obtain fold expression results.

\section{Cell viability assay}

Cell viability and proliferation were measured using the Cell Proliferation Kit I (MTT; Roche, Basel, Switzerland) according to the manufacturer's instructions and the method described in previous studies (Jiang et al., 2020; Zhao et al., 2021, p. 5). First, the transfected cells were stabilized by trypsin digestion. Following this, the cells were centrifuged, collected, resuspended in a single-cell suspension, and seeded in 96-well plates at the density of $1 \times 10^{4}$ cells/well. The cell cultures were maintained. A multifunctional microplate reader was used to measure optical density (OD) values. Cell growth curves were plotted using OD as the vertical axis and time as the horizontal axis. 
212

213

214

215

216

217

218

219

220

221

222

223

224

225

226

227

228

229

230

231

232

233

234

\section{Statistics and analysis}

Statistical and visual analyses were performed using $\mathrm{R}$ software [version 3.6.0]. The measurement data were expressed as $\mathrm{x} \pm \mathrm{s}$ and compared using the $\mathrm{t}$-test; the count data were expressed as percentages and compared using the $\chi^{2}$ test. $\mathrm{P}<0.05$ was considered statistically significant.

\section{Results}

Construction of a regulatory network for MKX, related genes and compounds based on the STITCH database

We used data from the STITCH database (http://stitch.embl.de) to identify MKX-related compounds and proteins and constructed MKX-related gene and compound regulatory networks (Figure 1A). Compounds related to MKX are Pro-Try and arsenite. Proteins related to MKX include SCXA, PROX2, suppressor interacting 3 A/histone deacetylase 1 (SIN3A/HDAC1), PBX1, MYOD1, TCF15, and PBX4. The regulatory network has nine nodes and 13 edges. Based on these proteins, we completed the GO functional enrichment analysis. The signaling pathways in which the MKX-related proteins were found to be enriched are as figure 1B. These results suggest that MKX-related protein functions are enriched in biological processes of biological development and transcriptional regulation.

\section{MKX-related symptom network constructed using SymMap}

The SymMap database integrates TCM and MM data on drug treatments and their molecular mechanisms, as shown in Figure 2. Herbs, ingredients of active compounds, and TCM and MM symptoms are included in this network. The compound 17-beta-estradiol, which can be 
235 derived from three herbs (Moschus, Cervi cornu Pantotrichum, and Armeniacae Semen 236 Amarum), was found to be capable of targeting and binding to MKX. MKX was found to be

237 potentially associated with nine diseases, which in turn were associated with 42 symptoms 238 (Table 1). In summary, the network demonstrates both TCM and MM symptoms associated with 239 MKX.

240

Molecular docking validation of the compounds Pro-Tyr and 17-beta-estradiol for target binding of MKX

The predicted three-dimensional structure of MKX based on the ExPASy tool is shown in

Figure 3A. The structures of Pro-Tyr and 17-beta-estradiol that were obtained from the PubChem database are shown in Figure 3B. Molecular docking using the AutoDock Vina software revealed that Pro-Tyr and 17-beta-estradiol target binding of MKX were at different loci (Figure 3C and D).

\section{Stability of complexes and molecular dynamics analysis}

Pro-Tyr as well as Estradiol fluctuated less during the kinetic simulations, indicating that

251

252

253

254

255

256

257

they were both stably bound within the active pocket (Figure 4A, B). RMSF showed that Pro-Tyr could better reduce the flexibility of the protein, which implied that Pro-Tyr was more stable with the protein system (Figure 4C). As shown in Figure 5A and Figure 5B, both molecules were maintained at the same binding site after MD simulation analysis. The small molecule seems to be closer to the protein after MD simulation analysis, which indicates that both molecules bind stably to the target protein. The results of MM/GBSA calculations showed that Estradiol/protein binding free energy was $-17.57 \pm 2.97 \mathrm{kcal} / \mathrm{mol}$ and Pro-Tyr/Protein binding free energy was - 
$25813.79 \pm 2.68 \mathrm{kcal} / \mathrm{mol}$ (Table 2). The binding energy of Estradiol/protein is mainly contributed

259 by van der Waals interaction, and that of Pro-Tyr/Protein is mainly contributed by electrostatic 260 interaction.

261

262

263

264

265

266

267

268

269

270

271

272

273

274

275

276

277

278

279

280

\section{Identification and functional analysis of proteins that may regulate MKX}

Analysis of the OncoBinder database revealed a significant regulatory correlation between MKX and a series of proteins (TBP, HSP90A, SIN3A, TBL1X, TBL1XR1, HSP90AA1, HDAC1, ELAVL1, GTF2B, and GTF2A1) (Figure 6A). We performed the GO and KEGG pathway enrichment analysis to identify the functions of these proteins (Figure6B). The GO enrichment analysis revealed that the functions of these proteins were significantly enriched in protein deacylation, protein deacetylation, histone deacetylation, transcriptional repressor complex, histone deacetylase complex, transcription factor complex, repressing transcription factor binding, RNA polymerase II general transcription initiation factor activity, and RNA polymerase II repressing transcription factor binding. The KEGG enrichment analysis revealed that these proteins were significantly enriched in viral carcinogenesis, basal transcription factors, and the IL-17 signaling pathway. The results suggest that MKX and the proteins significantly associated with it play important roles in protein acetylation and transcriptional regulation.

By combining the results presented in Figures 1, 2, and 3, it may be stated that the compounds Pro-Tyr and 17-beta-estradiol may bind MKX and affect its interaction with SIN3A and HDAC1 (Figure 7A). The integrated GSEA based on the MSigDB collections gene set database revealed that the low expression of MKX was significantly associated with osteoblast differentiation, signaling by NOTCH4 and Hedgehog, and the Hedgehog signaling pathway enrichment demonstrated significant correlation (Figure 7B). We evaluated the proliferation of 
281

282

283

284

285

286

287

288

289

290

291

292

293

294

295

296

297

298

299

300

301

302 303

BM-MSCs after treatment with different doses of 17-beta-estradiol and observed an increase in the proliferation rate (Figure 7C). This could be attributed to the upregulation of the HDAC1SIN3A/bone morphogenetic protein (BMP) pathway-related RUNX2 mRNA expression by 17beta-estradiol (Figure 7D). The results of our study suggest that 17-beta-estradiol may upregulate the HDAC1-SIN3A/BMP pathway.

\section{Discussion}

By predicting the three-dimensional structure of MKX and simulating molecular docking, this study revealed that Pro-Tyr and 17-beta-estradiol could target and bind MKX.

The present study identified potential compounds that target and regulate MKX and explore their functional mechanisms. Based on data from the STITCH and OncoBinder databases, MKX showed a significant regulatory correlation with SIN3A/HDAC1. The proliferation of BM-MSCs increased after treatment with different doses of 17-beta-estradiol. This may be attributed to the upregulation of the HDAC1-SIN3A/BMP pathway-related RUNX2 mRNA expression by 17beta-estradiol (Kim \& Lassar, 2003). The results of our study suggest that 17-beta-estradiol may upregulate the HDAC1-SIN3A/BMP pathway.

As a metabolite, Pro-Tyr is a dipeptide composed of L-proline and L-tyrosine residues (Klein et al., 1991). As a steroidal sex hormone, estradiol is actively involved in bone metabolism and has a multifunctional regulatory role in many cell types, including BM-MSCs. A recent study showed that supplementation with 17-beta-estradiol was effective in stimulating the proliferative capacity of human and mouse BM-MSCs. In addition, 17-beta-estradiol is known to increase the differentiation potential of MSCs, including osteogenic and lipogenic differentiation. Furthermore, 17-beta-estradiol regulates cellular function through its action on the estrogen 
304

305

306

307

308

309

310

311

312

313

314

315

316

317

318

319

320

321

322

323

324

325

326

receptor. Estrogen has been reported to increase osteogenic differentiation of bone marrow cells by upregulating osteogenic growth factors, including BMP-2, BMP-6, and transforming growth factor-beta 1 (TGF-beta 1) (Sammons et al., 2004). Estradiol has been reported to enhance the BMP-induced expression of RUNX2 (Matsumoto et al., 2010). SIN3A serves as a support component of the chromatin repression complex SIN3/HDAC (Zhao et al., 2019). Previous studies have demonstrated that SIN3A promotes the transcriptional repressor activity of Nkx3.2, thereby permitting BMP signaling and induction of somatic chondrogenesis by blocking the expression of anti-chondrogenic genes (Kim \& Lassar, 2003). HDAC is involved in regulating the differentiation of stem cells and osteoblasts (McGee-Lawrence \& Westendorf, 2011). Dynamic activities in the skeletal microenvironment require and contribute to rapid and temporal changes in gene expression within the cells responsible for the maintenance of bone cells (osteoblasts, osteoclasts, and osteocytes). The presence of HDAC1 in the promoter region of osteogenic genes is lower in differentiated osteoblasts. Numerous studies have reported that HDAC1 plays a role in the inhibition of osteoblast differentiation.

MKX, also known as Irxl (Iroquois homeobox-like 1) (Liu et al., 2010), is a member of the triamino-acid loop superfamily. MKX is necessary during the development of many tissues and organs in the cell proliferation, differentiation, and other processes (Selleri et al., 2001; Brendolan et al., 2005; Moens \& Selleri, 2006; van Tuyl et al., 2006; dilorio et al., 2007). MKX exerts a transcriptional repressive activity by recruiting the SIN3A/HDAC repressor complex (Bilioni et al., 2005; Anderson et al., 2009). In the present study, the results of GO and KEGG pathway enrichment analysis showed that MKX and its associated proteins play important functions in protein acetylation and transcriptional regulation and are closely involved in the biological processes of development and transcriptional regulation. These findings are consistent 
327 with those reported in previous studies. An integrated GSEA based on the MSigDB collections

328 gene set database revealed that high MKX expression inhibited osteogenesis.

329 Based on the existing literature and the results of the present study, it is reasonable to 330 speculate that the compounds Pro-Tyr and 17-beta-estradiol may bind MKX and competitively 331 inhibit the binding of MKX to SIN3A/HDAC1. This could interfere with the osteogenic 332 inhibitory effect of MKX, thereby promoting osteogenesis, which in turn manifests as HO during 333 tendon healing.

334 By predicting the three-dimensional structure of MKX and simulating molecular docking, 335 we found that Pro-Tyr and 17-beta-estradiol could target MKX. Based on data from the STITCH 336 and OncoBinder databases, we identified a protein, SIN3A/HDAC1, with significant regulatory relevance to MKX. The results of GO and KEGG pathway enrichment analysis suggested that the functions of MKX and its associated proteins were mainly enriched in osteogenic pathways. The compounds Pro-Tyr and 17-beta-estradiol may bind MKX and affect its interaction with SIN3A/HDAC1. This study initially revealed the interaction of MKX and its associated proteins with the small molecules, Pro-Tyr and 17-beta-estradiol, and the potential mechanism of HO. It provides new targets and ideas for the future development of novel anti-HO drugs. However, the present study was limited to bioinformatics analysis, and the exact mechanism of action of MKX, Pro-Tyr, 17-beta-estradiol, and SIN3A/HDAC1 remains unclear. Future antagonist and animal model experiments are necessary to validate the mechanisms.

\section{Conclusions}

This study initially revealed the interaction of MKX and its associated proteins with the 349 small molecules, Pro-Tyr and 17-beta-estradiol, and the potential mechanism of HO. It provides 
350 new targets and ideas for the future development of novel anti-HO drugs.

351

352 Funding

353 This study did not receive any funding.

354

355 Data access

356 All raw data of this study have been uploaded.

357

358 Acknowledgements

359 We would like to thank the anonymous reviewers for their valuable comments on our revisions.

360 We thank Bullet Edits Limited for the linguistic editing and proofreading of the manuscript.

361

362 Competing interests

363 No comping interests.

364

365

Authors' contributions

YZ, JZ and FW came up with and designed the experiments. YZ, JZ and FW conducted the

experiments although all authors analyzed the data. In addition, YZ, JZ, CS and FW contributed 368 the reagents/materials/analysis tools. $\mathrm{YZ}$ and $\mathrm{FW}$ wrote the manuscript. All authors contributed to the article and approved the submitted version.

370

371 
372

373

374

375

376

377

378

379

380

381

382

383

384

385

386

387

388

389

390

391

392

393

394

395

396

397

398

399

400

401

402

403

404

405

406

407

408

409

410

411

\section{References}

Anderson DM, Beres BJ, Wilson-Rawls J, Rawls A. 2009. The homeobox gene Mohawk represses transcription by recruiting the $\sin 3 \mathrm{~A} / \mathrm{HDAC}$ co-repressor complex. Developmental Dynamics: An Official Publication of the American Association of Anatomists 238:572-580. DOI: 10.1002/dvdy.21873.

Bilioni A, Craig G, Hill C, McNeill H. 2005. Iroquois transcription factors recognize a unique motif to mediate transcriptional repression in vivo. Proceedings of the National Academy of Sciences of the United States of America 102:14671-14676. DOI: 10.1073/pnas.0502480102.

Brendolan A, Ferretti E, Salsi V, Moses K, Quaggin S, Blasi F, Cleary ML, Selleri L. 2005. A $\mathrm{Pbx} 1$-dependent genetic and transcriptional network regulates spleen ontogeny. Development (Cambridge, England) 132:3113-3126. DOI: 10.1242/dev.01884.

Chen Y, Zheng Y, Fong P, Mao S, Wang Q. 2020. The application of the MM/GBSA method in the binding pose prediction of FGFR inhibitors. Physical Chemistry Chemical Physics 22:96569663. DOI: 10.1039/D0CP00831A.

diIorio P, Alexa K, Choe S-K, Etheridge L, Sagerström CG. 2007. TALE-family homeodomain proteins regulate endodermal sonic hedgehog expression and pattern the anterior endoderm. Developmental Biology 304:221-231. DOI: 10.1016/j.ydbio.2006.12.024.

Friesner RA, Banks JL, Murphy RB, Halgren TA, Klicic JJ, Mainz DT, Repasky MP, Knoll EH, Shelley M, Perry JK, Shaw DE, Francis P, Shenkin PS. 2004. Glide: A New Approach for Rapid, Accurate Docking and Scoring. 1. Method and Assessment of Docking Accuracy. Journal of Medicinal Chemistry 47:1739-1749. DOI: 10.1021/jm0306430.

Gasteiger E. 2003. ExPASy: the proteomics server for in-depth protein knowledge and analysis. Nucleic Acids Research 31:3784-3788. DOI: 10.1093/nar/gkg563.

Genheden S, Ryde U. 2015. The MM/PBSA and MM/GBSA methods to estimate ligand-binding affinities. Expert Opinion on Drug Discovery 10:449-461. DOI: 10.1517/17460441.2015.1032936.

Giddings VL, Beaupr?? GS, Whalen RT, Carter DR. 2000. Calcaneal loading during walking and running: Medicine \& Science in Sports \& Exercise 32:627-634. DOI: 10.1097/00005768200003000-00012.

He D, Huang J, Zhang Z, Du Q, Peng W, Yu R, Zhang S, Zhang S, Qin Y. 2019. A Network Pharmacology-Based Strategy For Predicting Active Ingredients And Potential Targets Of LiuWei DiHuang Pill In Treating Type 2 Diabetes Mellitus. Drug Design, Development and Therapy Volume 13:3989-4005. DOI: 10.2147/DDDT.S216644.

Ito Y, Toriuchi N, Yoshitaka T, Ueno-Kudoh H, Sato T, Yokoyama S, Nishida K, Akimoto T, Takahashi M, Miyaki S, Asahara H. 2010. The Mohawk homeobox gene is a critical regulator of tendon differentiation. Proceedings of the National Academy of Sciences 107:10538-10542. DOI: 10.1073/pnas.1000525107.

James R, Kesturu G, Balian G, Chhabra AB. 2008. Tendon: Biology, Biomechanics, Repair, Growth Factors, and Evolving Treatment Options. The Journal of Hand Surgery 33:102-112. DOI: 10.1016/j.jhsa.2007.09.007. 
412 Jiang Y, Zhou J, Zhao J, Zhang H, Li L, Li H, Chen L, Hu J, Zheng W, Jing Z. 2020. The $413 \mathrm{U} 2 \mathrm{AF} 2$ /circRNA ARF1/miR-342-3p/ISL2 feedback loop regulates angiogenesis in glioma stem 414 cells. Journal of Experimental \& Clinical Cancer Research 39:182. DOI: 10.1186/s13046-020415 01691-y.

416 Kim D-W, Lassar AB. 2003. Smad-dependent recruitment of a histone deacetylase/Sin3A 417 complex modulates the bone morphogenetic protein-dependent transcriptional repressor activity 418 of Nkx3.2. Molecular and Cellular Biology 23:8704-8717. DOI: 10.1128/MCB.23.23.87044198717.2003.

420 Kimura W, Machii M, Xue X, Sultana N, Hikosaka K, Sharkar MTK, Uezato T, Matsuda M, 421 Koseki H, Miura N. 2011. Irxl1 mutant mice show reduced tendon differentiation and no 422 patterning defects in musculoskeletal system development. genesis 49:2-9. DOI: $423 \quad 10.1002 / \mathrm{dvg} .20688$.

424 Klein CL, Cobbinah I, Rouselle D, Malmstrom MC, Stevens ED. 1991. Structure of L-prolyl-L425 tyrosine monohydrate. Acta Crystallographica. Section C, Crystal Structure Communications 47 426 ( Pt 11):2386-2388. DOI: 10.1107/s0108270191005760.

427 Larini L, Mannella R, Leporini D. 2007. Langevin stabilization of molecular-dynamics 428 simulations of polymers by means of quasisymplectic algorithms. The Journal of Chemical 429 Physics 126:104101. DOI: 10.1063/1.2464095.

430 Lee T-S, Allen BK, Giese TJ, Guo Z, Li P, Lin C, McGee TD, Pearlman DA, Radak BK, Tao Y, 431 Tsai H-C, Xu H, Sherman W, York DM. 2020. Alchemical Binding Free Energy Calculations in 432 AMBER20: Advances and Best Practices for Drug Discovery. Journal of Chemical Information 433 and Modeling 60:5595-5623. DOI: 10.1021/acs.jcim.0c00613.

434 Liu W, Watson SS, Lan Y, Keene DR, Ovitt CE, Liu H, Schweitzer R, Jiang R. 2010. The 435 Atypical Homeodomain Transcription Factor Mohawk Controls Tendon Morphogenesis. 436 Molecular and Cellular Biology 30:4797-4807. DOI: 10.1128/MCB.00207-10.

437 Liu H, Xu J, Jiang R. 2019. Mkx -Deficient Mice Exhibit Hedgehog Signaling-Dependent 438 Ectopic Ossification in the Achilles Tendons. Journal of Bone and Mineral Research 34:557439 569. DOI: 10.1002/jbmr.3630.

440 Liu H, Zhang C, Zhu S, Lu P, Zhu T, Gong X, Zhang Z, Hu J, Yin Z, Heng BC, Chen X, Wei 441 Ouyang H. 2015. Mohawk Promotes the Tenogenesis of Mesenchymal Stem Cells Through 442 Activation of the TGF $\beta$ Signaling Pathway: Mkx Promotes the Tenogenesis of MSCs. STEM 443 CELLS 33:443-455. DOI: 10.1002/stem.1866.

444 Maier JA, Martinez C, Kasavajhala K, Wickstrom L, Hauser KE, Simmerling C. 2015. ff14SB: 445 Improving the Accuracy of Protein Side Chain and Backbone Parameters from ff99SB. Journal 446 of Chemical Theory and Computation 11:3696-3713. DOI: 10.1021/acs.jctc.5b00255.

447 Matsumoto Y, Otsuka F, Takano M, Mukai T, Yamanaka R, Takeda M, Miyoshi T, Inagaki K, 448 Sada K, Makino H. 2010. Estrogen and glucocorticoid regulate osteoblast differentiation 449 through the interaction of bone morphogenetic protein-2 and tumor necrosis factor-alpha 450 in $\mathrm{C} 2 \mathrm{C} 12$ cells. Molecular and Cellular Endocrinology 325:118-127. DOI: 451 10.1016/j.mce.2010.05.004.

452 McGee-Lawrence ME, Westendorf JJ. 2011. Histone deacetylases in skeletal development and 
453 bone mass maintenance. Gene 474:1-11. DOI: 10.1016/j.gene.2010.12.003.

454 Mehta P, Miszta P, Filipek S. 2021. Molecular Modeling of Histamine Receptors-Recent 455 Advances in Drug Discovery. Molecules 26:1778. DOI: 10.3390/molecules26061778.

456 Moens CB, Selleri L. 2006. Hox cofactors in vertebrate development. Developmental Biology 457 291:193-206. DOI: 10.1016/j.ydbio.2005.10.032.

458 Nguyen H, Roe DR, Simmerling C. 2013. Improved Generalized Born Solvent Model 459 Parameters for Protein Simulations. Journal of Chemical Theory and Computation 9:2020-2034. 460 DOI: $10.1021 /$ ct3010485.

461 O'Brien EJO, Frank CB, Shrive NG, Hallgrímsson B, Hart DA. 2012. Heterotopic mineralization 462 (ossification or calcification) in tendinopathy or following surgical tendon trauma. International 463 Journal of Experimental Pathology 93:319-331. DOI: 10.1111/j.1365-2613.2012.00829.x.

464 Sammons J, Ahmed N, El-Sheemy M, Hassan HT. 2004. The role of BMP-6, IL-6, and BMP-4 465 in mesenchymal stem cell-dependent bone development: effects on osteoblastic differentiation 466 induced by parathyroid hormone and vitamin D(3). Stem Cells and Development 13:273-280. 467 DOI: $10.1089 / 154732804323099208$.

468 Selleri L, Depew MJ, Jacobs Y, Chanda SK, Tsang KY, Cheah KS, Rubenstein JL, O’Gorman S, 469 Cleary ML. 2001. Requirement for Pbx1 in skeletal patterning and programming chondrocyte proliferation and differentiation. Development (Cambridge, England) 128:3543-3557. Subramanian A, Tamayo P, Mootha VK, Mukherjee S, Ebert BL, Gillette MA, Paulovich A, Pomeroy SL, Golub TR, Lander ES, Mesirov JP. 2005. Gene set enrichment analysis: A knowledge-based approach for interpreting genome-wide expression profiles. Proceedings of the National Academy of Sciences 102:15545-15550. DOI: 10.1073/pnas.0506580102. Suzuki H, Ito Y, Shinohara M, Yamashita S, Ichinose S, Kishida A, Oyaizu T, Kayama T, Nakamichi R, Koda N, Yagishita K, Lotz MK, Okawa A, Asahara H. 2016. Gene targeting of the transcription factor Mohawk in rats causes heterotopic ossification of Achilles tendon via failed tenogenesis. Proceedings of the National Academy of Sciences 113:7840-7845. DOI: 10.1073/pnas.1522054113.

Szklarczyk D, Santos A, von Mering C, Jensen LJ, Bork P, Kuhn M. 2016. STITCH 5: augmenting protein-chemical interaction networks with tissue and affinity data. Nucleic Acids Research 44:D380-D384. DOI: 10.1093/nar/gkv1277. van Tuyl M, Liu J, Groenman F, Ridsdale R, Han RNN, Venkatesh V, Tibboel D, Post M. 2006. Iroquois genes influence proximo-distal morphogenesis during rat lung development. American Journal of Physiology. Lung Cellular and Molecular Physiology 290:L777-L789. DOI: 10.1152/ajplung.00293.2005.

487 Van Coillie S, Liang L, Zhang Y, Wang H, Fang J-Y, Xu J. 2016. OncoBinder facilitates interpretation of proteomic interaction data by capturing coactivation pairs in cancer. Oncotarget 7:17608-17615. DOI: 10.18632/oncotarget.7305.

Wang J, Wolf RM, Caldwell JW, Kollman PA, Case DA. 2004. Development and testing of a general amber force field. Journal of Computational Chemistry 25:1157-1174. DOI:

493 Wu Y, Zhang F, Yang K, Fang S, Bu D, Li H, Sun L, Hu H, Gao K, Wang W, Zhou X, Zhao Y, 
494 Chen J. 2019. SymMap: an integrative database of traditional Chinese medicine enhanced by 495 symptom mapping. Nucleic Acids Research 47:D1110-D1117. DOI: 10.1093/nar/gky1021.

496 Yu G, Wang L-G, Han Y, He Q-Y. 2012. clusterProfiler: an R Package for Comparing 497 Biological Themes Among Gene Clusters. OMICS: A Journal of Integrative Biology 16:284-287. 498 DOI: 10.1089/omi.2011.0118.

499 Zhao J, Jiang Y, Zhang H, Zhou J, Chen L, Li H, Xu J, Zhang G, Jing Z. 2021. The 500 SRSF1/circATP5B/miR-185-5p/HOXB5 feedback loop regulates the proliferation of glioma 501 stem cells via the IL6-mediated JAK2/STAT3 signaling pathway. Journal of Experimental \& 502 Clinical Cancer Research 40:134. DOI: 10.1186/s13046-021-01931-9.

503 Zhao P, Li S, Wang H, Dang Y, Wang L, Liu T, Wang S, Li X, Zhang K. 2019. Sin3a regulates 504 the developmental progression through morula-to-blastocyst transition via Hdac1. FASEB 505 journal: official publication of the Federation of American Societies for Experimental Biology 506 33:12541-12553. DOI: 10.1096/fj.201901213R. 
507

508

509

510

511

512

513

514

515

516

517

518

519

520

521

522

523

524

525

526

527

528

529

\section{Figure Legends}

Figure 1. Analysis of MKX-related gene and compound regulatory networks (A) Construction of MKX, related genes, and compound regulatory networks based on data from the STITCH database; (B) The GO function analysis of genes in the subnetwork.

\section{Figure 2. MKX-related symptom network built with SymMap.}

Figure 3. Pro-Tyr and 17-beta-estradiol can target and bind MKX (A) The predicted threedimensional structure of MKX based on the ExPASy tool; (B) Two-dimensional structures of Pro-Tyr and 17-beta-estradiol; (C-D) Schematic representation of Pro-Tyr and 17-beta-estradiol targeting MKX binding at different loci.

Figure 4. The stability of the complexes as well as the binding energy. To simulate the fluctuation of small molecules or proteins in the process, expressed as root mean square deviation (RMSD) (A, B); Changes in the flexibility of the protein in the presence of different ligand binding $(\mathrm{C})$.

Figure 5. Protein conformation before and after MD. 17-beta-estradiol/MKX protein complex (A) and Pro-Tyr/MKX protein complex (B). Cyan color indicates pre-MD mimic conformation and green color indicates post-MD mimic conformation.

Figure 6. Identification and enrichment analysis of proteins that may regulate MKX. (A) The "co-activation" scores of candidate MKX-associated proteins are plotted in the lower panel, and the lines between the proteins indicate a statistical relationship between them $(\mathrm{P}<0.05)$; (B) The GO function and KEGG pathway enrichment analysis of the MKX-related proteins. Analysis of the potential functions of MKX and 17-beta-estradiol for osteogenic differentiation Figure 7. Analysis of the potential functions of MKX (A) Interrelationship between Pro-Tyr, 17-beta-estradiol, MKX, SIN3A, and HDAC1; (B) GSEA of MKX; (C) Proliferation of BM- 
530 MSCs after treatment with different doses of 17-beta-estradiol; (D) Effect of different doses of 531 17-beta-estradiol on RUNX2 mRNA expression 
Figure 1

Analysis of MKX-related gene and compound regulatory networks.

(A) Construction of MKX, related genes, and compound regulatory networks based on data from the STITCH database; (B) The GO function analysis of genes in the subnetwork.

A.

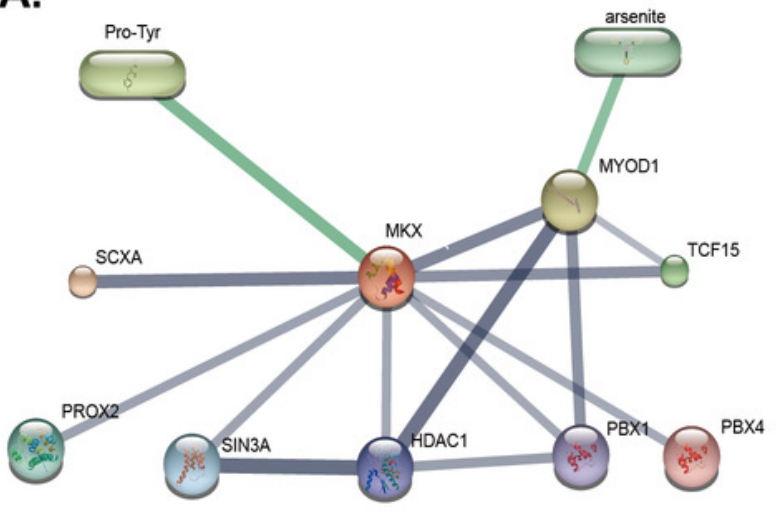

Number of nodes: 9

Number of edges: 13

Average node degree: 2.89

Clustering coefficient: $\mathbf{0 . 8 3 5}$

Expected number of edges: 9

PPI enrichment p-value: $\mathbf{0 . 1 2 8}$

B.
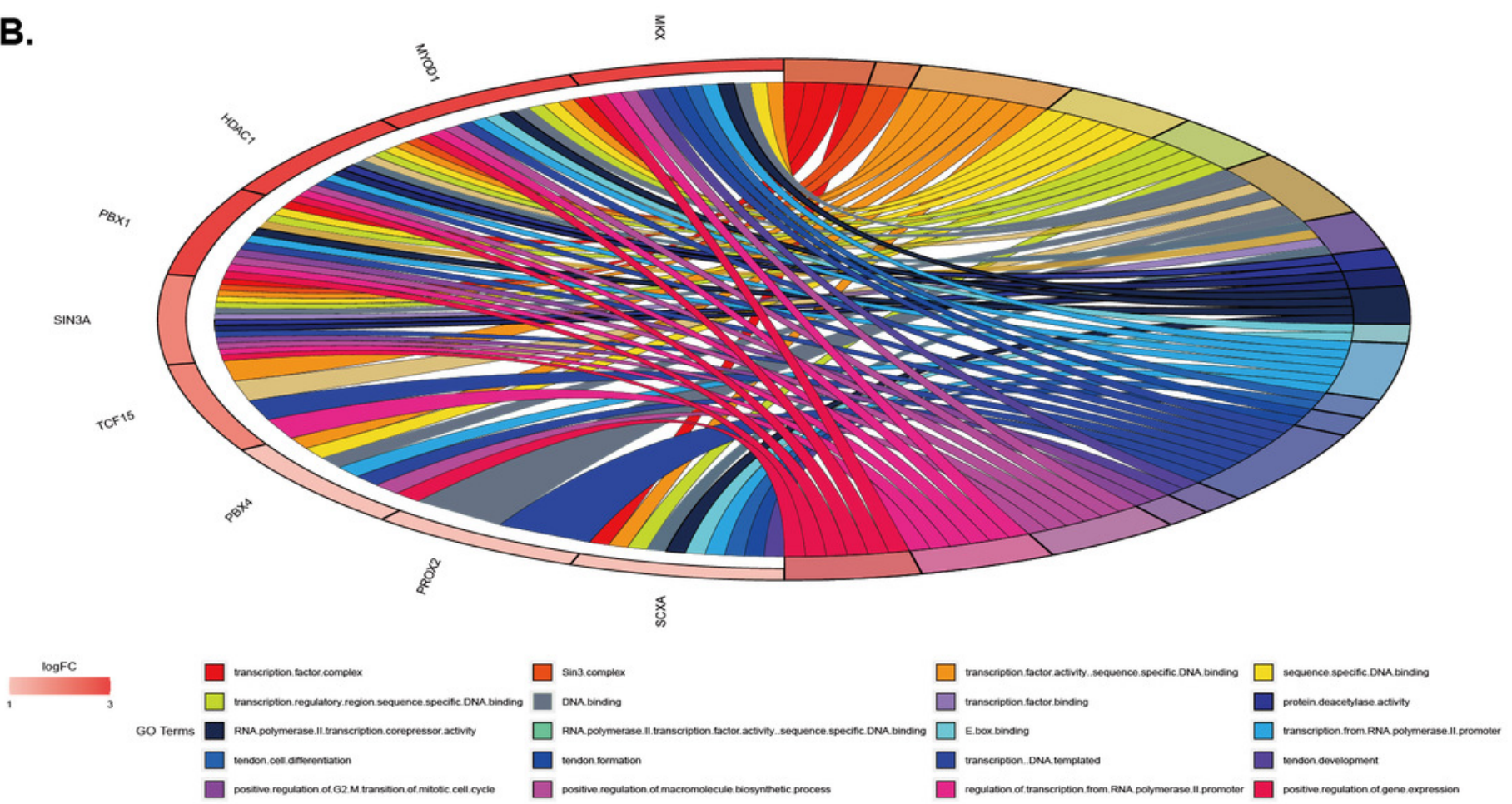
Figure 2

MKX-related symptom network built with SymMap.

\section{MKX-related symptom network built with SymMap.}

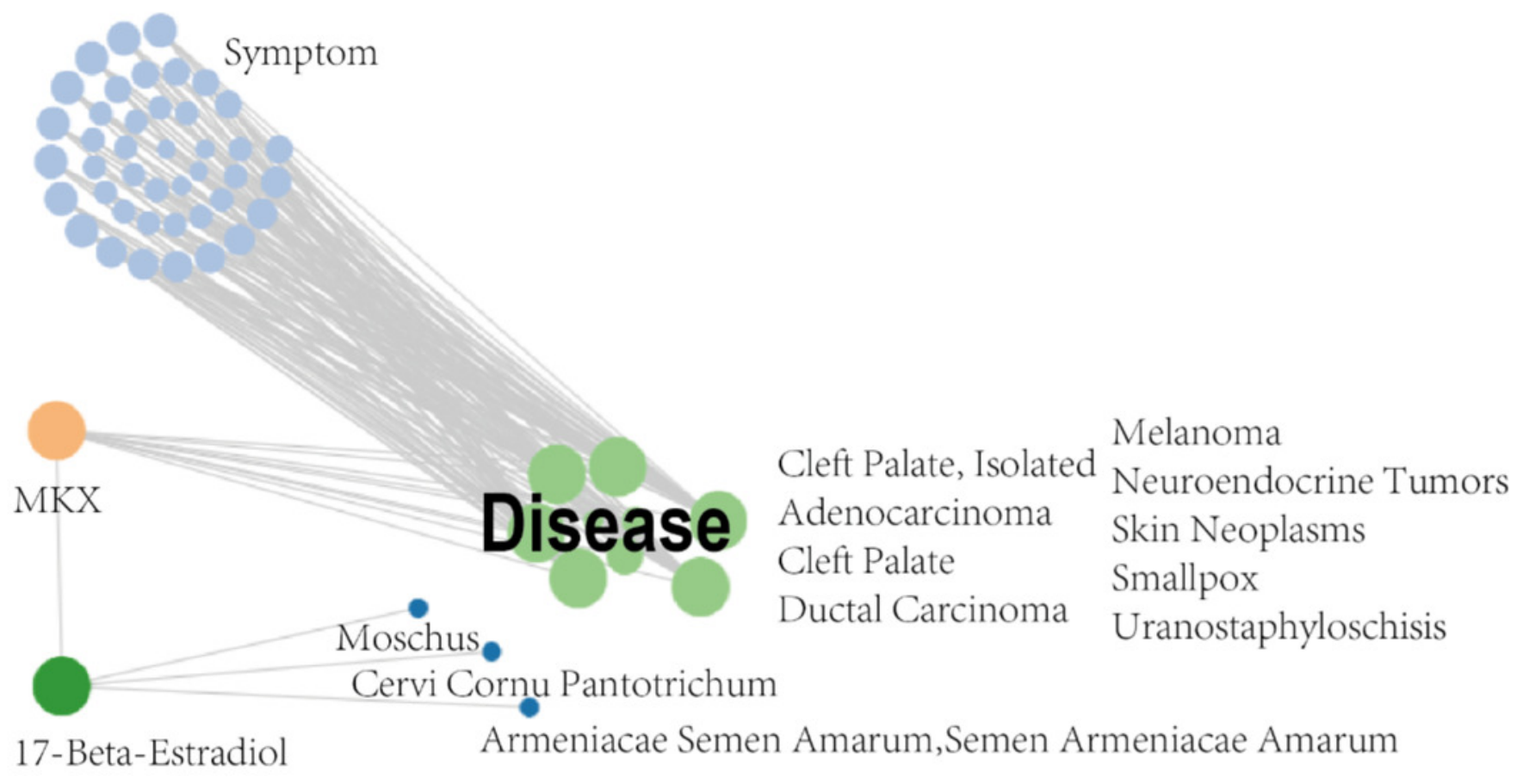


Figure 3

Pro-Tyr and 17-beta-estradiol can target and bind MKX.

The predicted three-dimensional structure of MKX based on the ExPASy tool; (B) Twodimensional structures of Pro-Tyr and 17-beta-estradiol; (C-D) Schematic representation of Pro-Tyr and 17-beta-estradiol targeting MKX binding at different loci.

A.

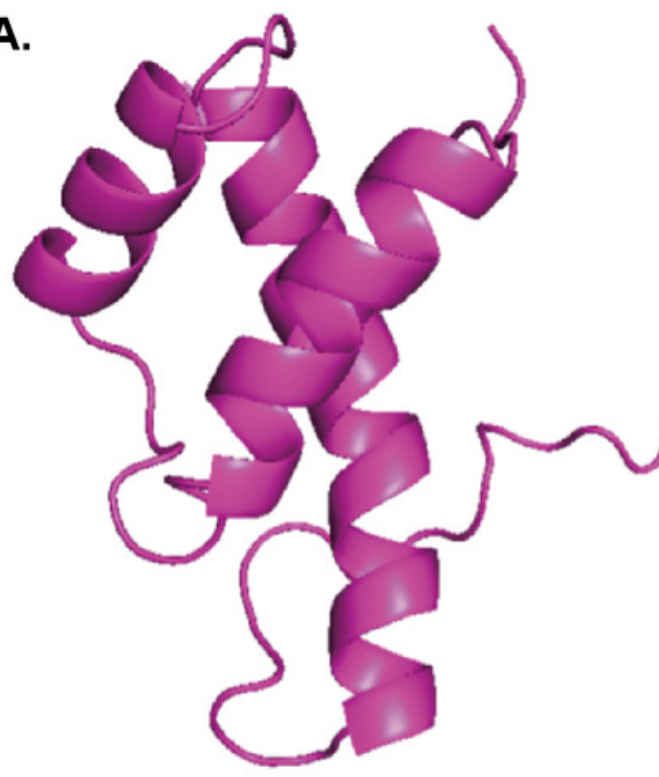

C.

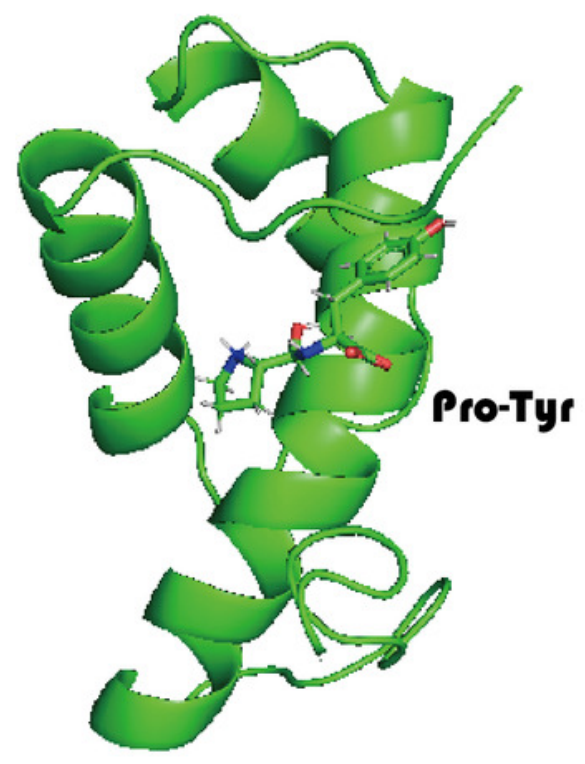

B.

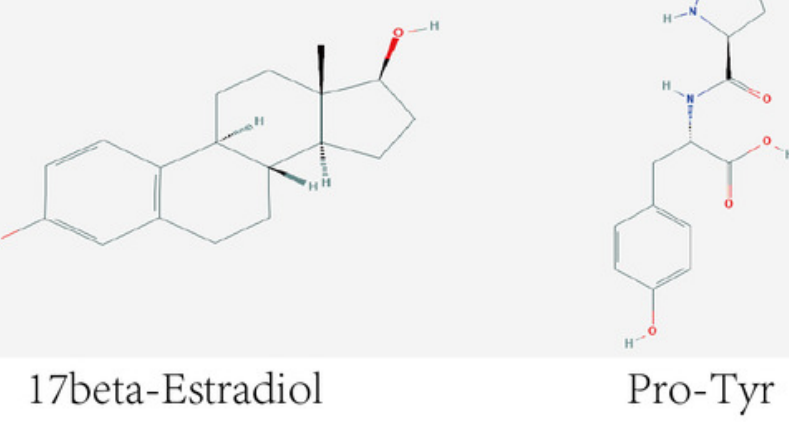

D.

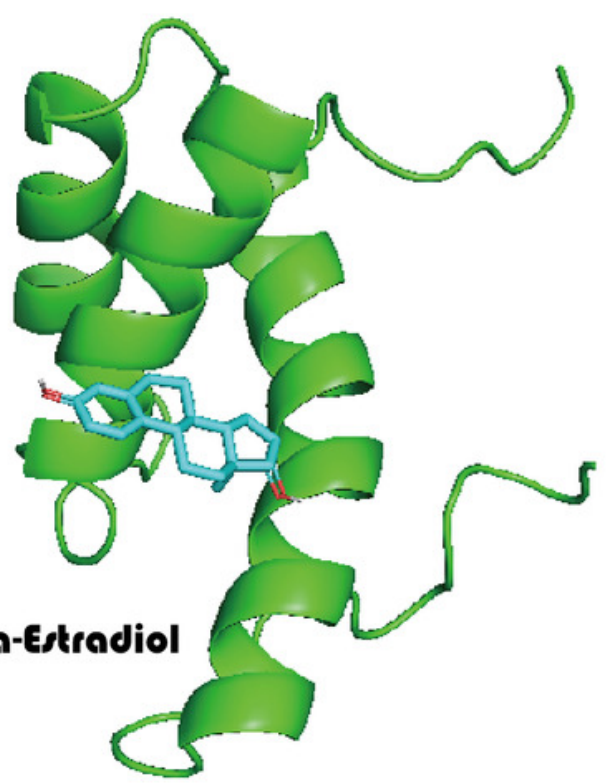


Figure 4

The stability of the complexes as well as the binding energy.

To simulate the fluctuation of small molecules or proteins in the process, expressed as root mean square deviation (RMSD) (A, B); Changes in the flexibility of the protein in the presence of different ligand binding (C).
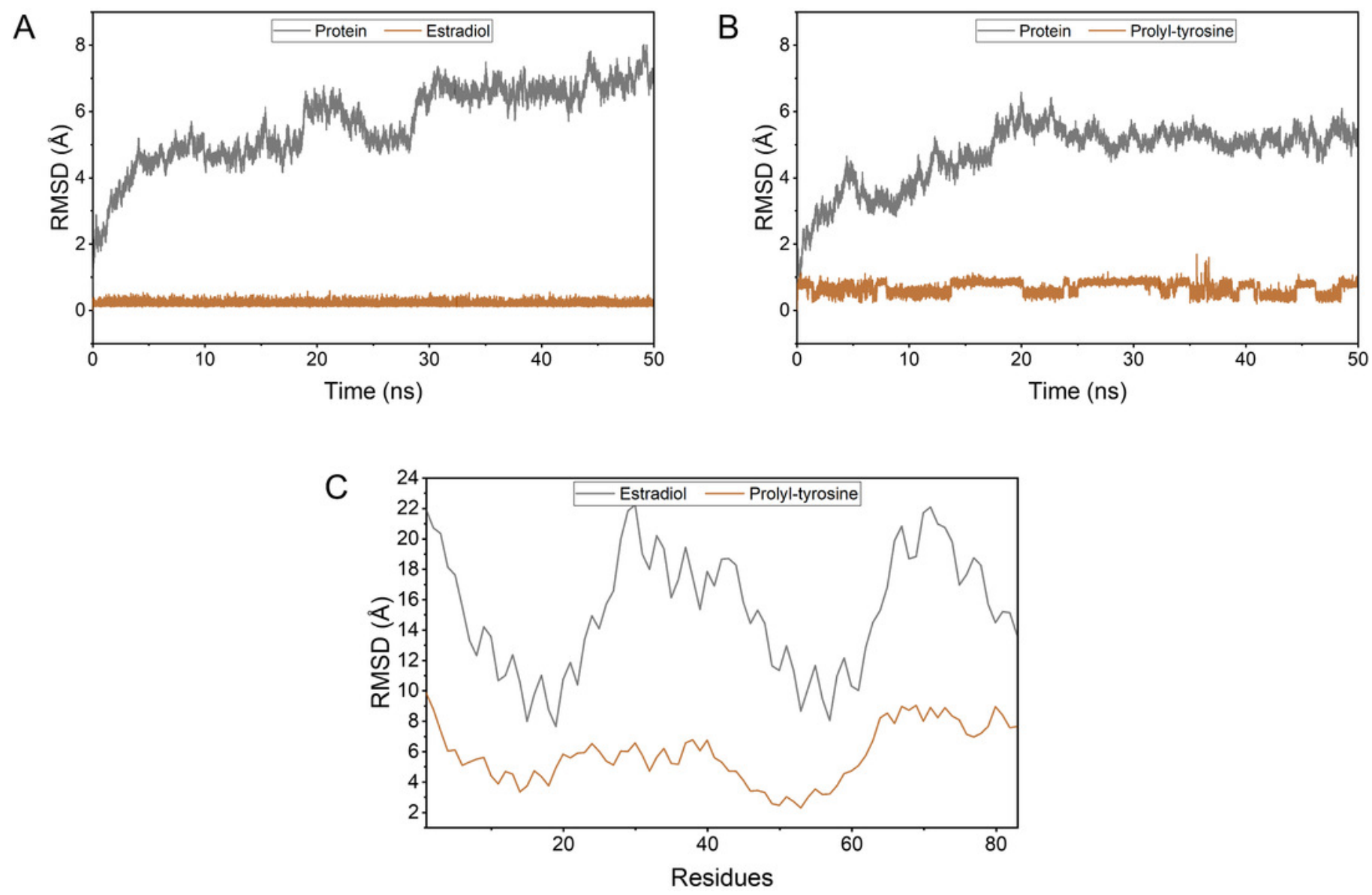
Figure 5

Protein conformation before and after MD.

17-beta-estradiol/MKX protein complex (A) and Pro-Tyr/MKX protein complex (B). Cyan color indicates pre-MD mimic conformation and green color indicates post-MD mimic conformation.

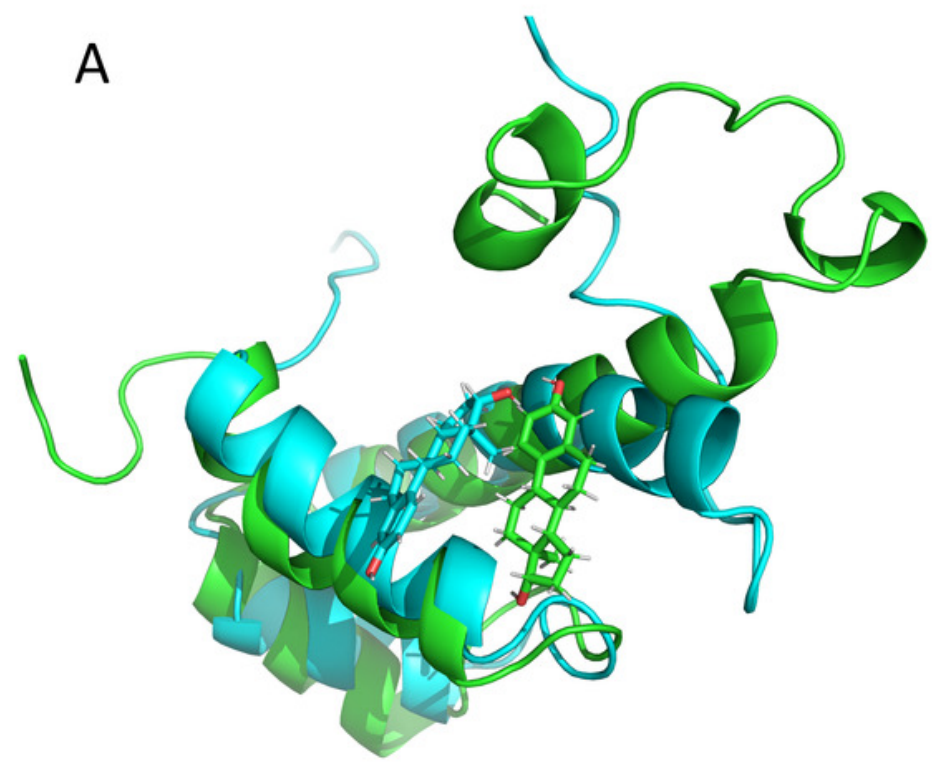

Estradiol/protein complex

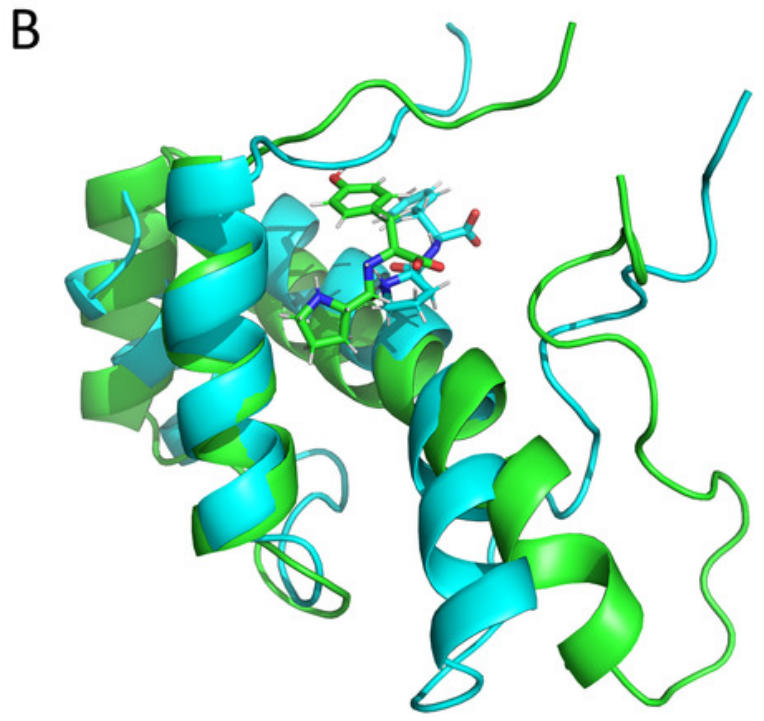

Prolyl-tyrosine/protein complex 


\section{Figure 6}

Identification and enrichment analysis of proteins that may regulate MKX.

(A) The "co-activation" scores of candidate MKX-associated proteins are plotted in the lower panel, and the lines between the proteins indicate a statistical relationship between them ( $P$ $<0.05)$; (B) The GO function and KEGG pathway enrichment analysis of the MKX-related proteins. Analysis of the potential functions of MKX and 17-beta-estradiol for osteogenic differentiation

A.

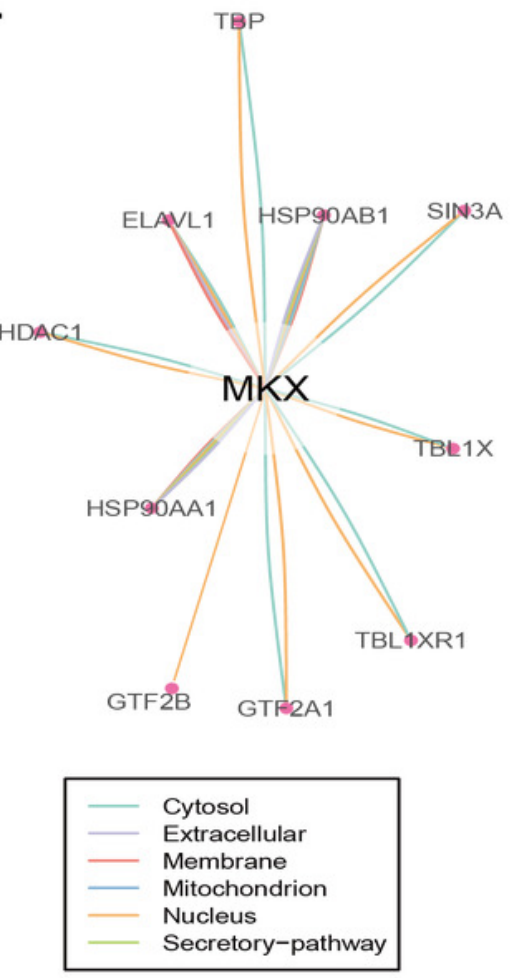

B.

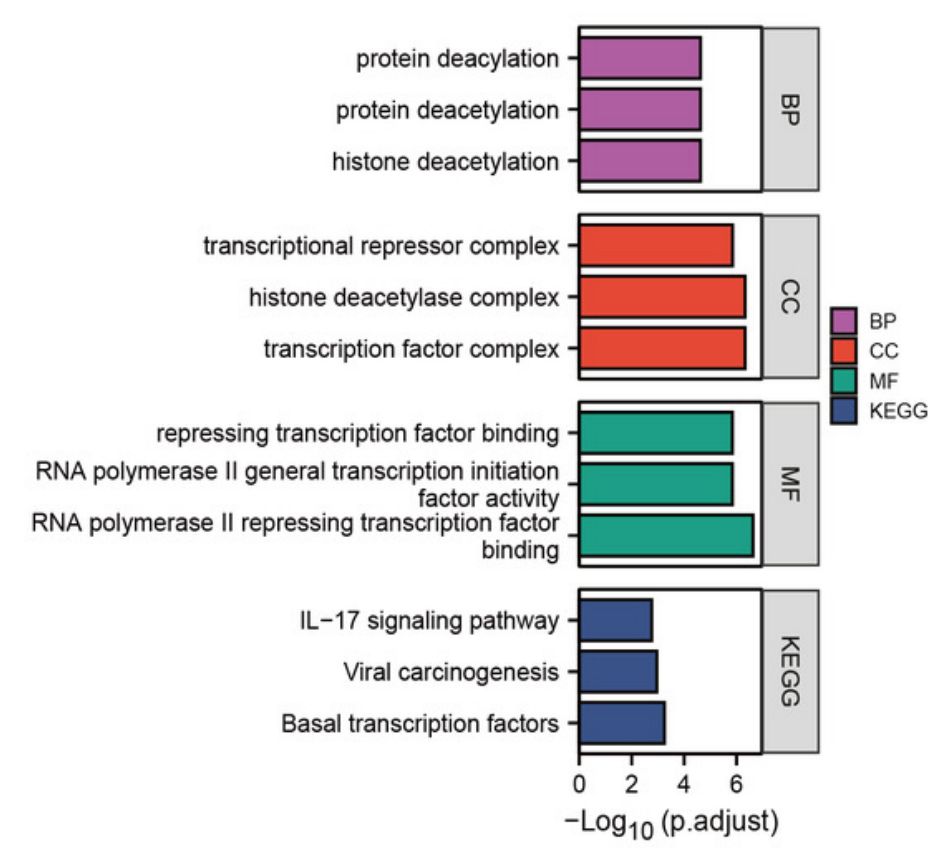


Figure 7

Analysis of the potential functions of MKX.

(A) Interrelationship between Pro-Tyr, 17-beta-estradiol, MKX, SIN3A, and HDAC1; (B) GSEA of MKX; (C) Proliferation of BM-MSCs after treatment with different doses of 17-beta-estradiol; (D) Effect of different doses of 17-beta-estradiol on RUNX2 mRNA expression 
A.

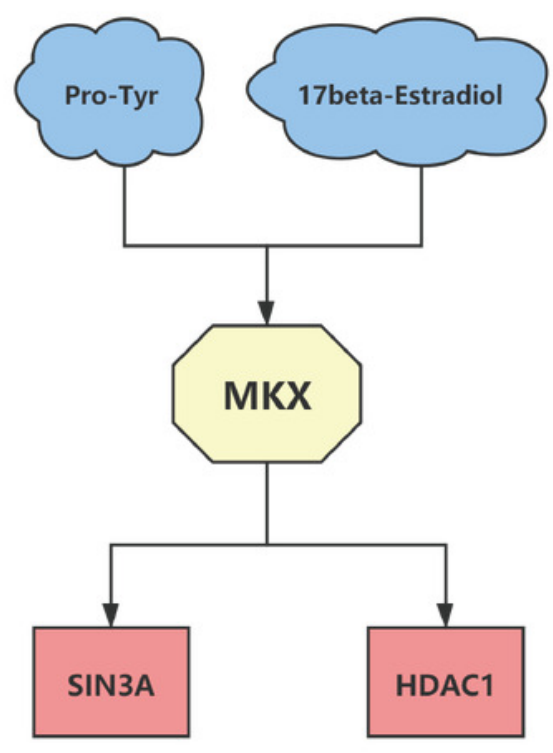

c.

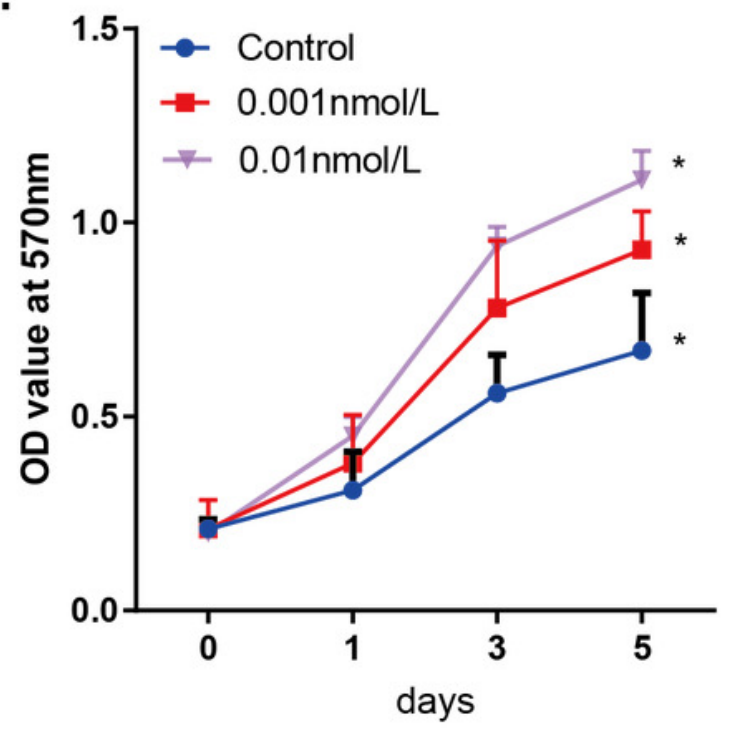

B.

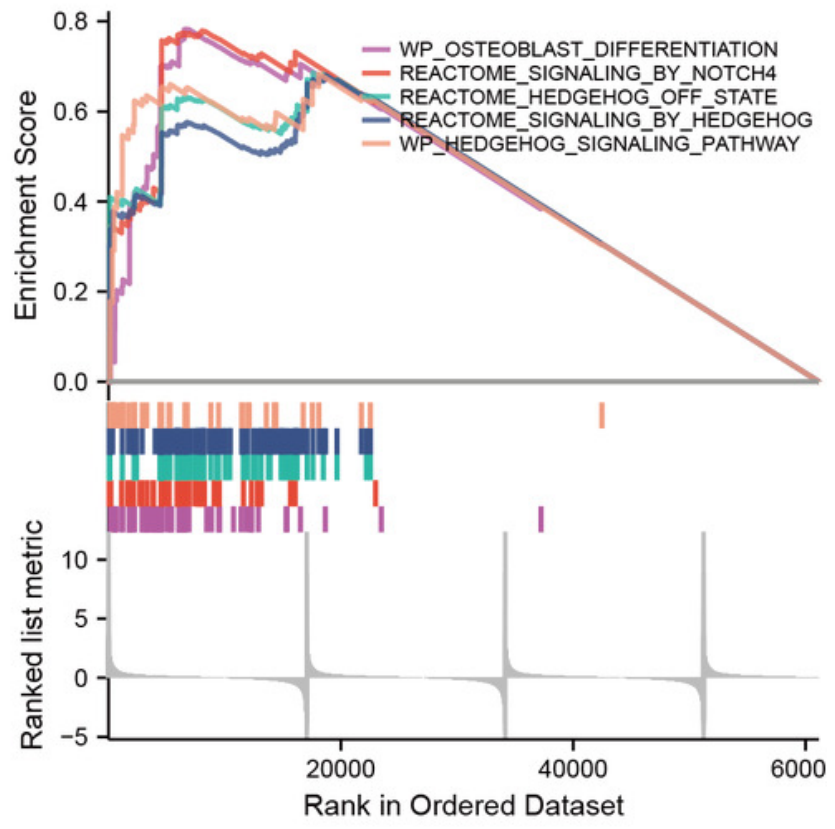

D.

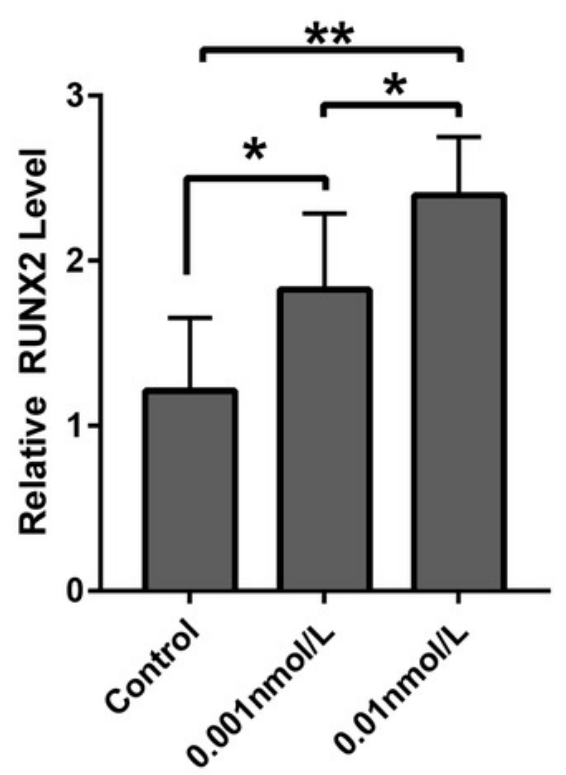


Table $\mathbf{1}$ (on next page)

Potential symptoms related with MKX.

Potential symptoms related with MKX. 
Table 1. Potential symptoms related with MKX.

\begin{tabular}{|c|c|c|}
\hline ID & Term & $P$ value \\
\hline SMMS00054 & Constipation & $p<0.001$ \\
\hline SMMS00096 & Hemoptysis & 0.039 \\
\hline SMMS00115 & Vertigo & 0.010 \\
\hline SMMS00132 & Hoarseness & $p<0.001$ \\
\hline SMMS00138 & Toothache & 0.002 \\
\hline SMMS00223 & Chest Pain & 0.008 \\
\hline SMMS00225 & Dyspnea & $p<0.001$ \\
\hline SMMS00233 & Edema & 0.001 \\
\hline SMMS00236 & Muscle Weakness & $p<0.001$ \\
\hline SMMS00261 & Trismus & $p<0.001$ \\
\hline SMMS00279 & Profound Mental Retardation & $p<0.001$ \\
\hline SMMS00327 & Emesis & 0.006 \\
\hline SMMS00329 & Crescendo Angina & $p<0.001$ \\
\hline SMMS00332 & Jaundice & $p<0.001$ \\
\hline SMMS00349 & Arthralgia & $p<0.001$ \\
\hline SMMS00369 & Polyuria & $p<0.001$ \\
\hline SMMS00379 & Paralysis & 0.001 \\
\hline SMMS00403 & Cough & $p<0.001$ \\
\hline SMMS00415 & Nausea & $p<0.001$ \\
\hline SMMS00441 & Skin Pruritus & 0.010 \\
\hline SMMS00457 & Spasm & 0.026 \\
\hline SMMS00461 & Paresis & $p<0.001$ \\
\hline SMMS00522 & Dysphonia & $p<0.001$ \\
\hline SMMS00540 & Angina Pectoris & 0.001 \\
\hline SMMS00549 & Facial Paralysis & 0.006 \\
\hline SMMS00553 & Hemiplegia & $p<0.001$ \\
\hline SMMS00577 & Musculoskeletal Pain & 0.002 \\
\hline SMMS00629 & Hyperemesis Gravidarum & $p<0.001$ \\
\hline SMMS00633 & Cyanosis & 0.001 \\
\hline SMMS00653 & Neuralgia & 0.002 \\
\hline SMMS00656 & Backache & $p<0.001$ \\
\hline SMMS00682 & Sleep Disorder & 0.006 \\
\hline SMMS00774 & Loss Of Appetite & 0.001 \\
\hline
\end{tabular}




\begin{tabular}{lll}
\hline SMMS00870 & Face Pain & $p<0.001$ \\
\hline SMMS00871 & Neck Pain & $p<0.001$ \\
\hline SMMS00901 & Purpura & 0.003 \\
\hline SMMS00928 & Headache & 0.002 \\
\hline SMMS00973 & Anorexia Symptom & 0.001 \\
\hline SMMS01036 & Fever Symptoms & $p<0.001$ \\
\hline SMMS01116 & Spasticity Muscle & $p<0.001$ \\
\hline SMMS01125 & Thrombocytopenic Purpura & 0.001 \\
\hline SMMS01143 & Vomiting & 0.003 \\
\hline
\end{tabular}

1 
Table 2 (on next page)

Table 2. Binding free energies and energy components predicted by MM/GBSA $(\mathrm{kcal} / \mathrm{mol})$.

Binding free energies and energy components predicted by MM/GBSA (kcal/mol). 
2 Table 2. Binding free energies and energy components predicted by MM/GBSA $3(\mathrm{kcal} / \mathrm{mol})$.

\begin{tabular}{ccc}
\hline System name & Estradiol/protein & Pro-Tyr/Protein \\
\hline$\Delta \boldsymbol{E}_{\mathrm{vdw}}$ & $-22.14 \pm 3.08$ & $-24.48 \pm 4.93$ \\
$\boldsymbol{\Delta} \boldsymbol{E}_{\text {elec }}$ & $-3.04 \pm 3.98$ & $-36.72 \pm 17.27$ \\
$\boldsymbol{\Delta} \mathbf{G}_{\mathrm{GB}}$ & $10.32 \pm 4.23$ & $51.05 \pm 15.29$ \\
$\boldsymbol{\Delta} \mathbf{G}_{\mathrm{SA}}$ & $-2.70 \pm 0.32$ & $-3.62 \pm 0.32$ \\
$\boldsymbol{\Delta} \mathbf{G}_{\text {bind }}$ & $-17.57 \pm 2.97$ & $-13.79 \pm 2.68$ \\
\hline
\end{tabular}

$4 \quad \Delta \mathrm{E}_{\mathrm{vd} w}$ : van der Waals energy.

$5 \Delta \mathrm{E}_{\text {elec: }}$ : electrostatic energy.

$6 \Delta \mathrm{G}_{\mathrm{GB}}$ : electrostatic contribution to solvation.

$7 \quad \Delta \mathrm{G}_{\mathrm{SA}}$ : non-polar contribution to solvation.

$8 \Delta \mathrm{G}_{\text {bind }}$ : binding free energy.

9 